\title{
Aquaporin 3 maintains the stemness of CD133+ hepatocellular carcinoma cells by activating STAT3
}

\author{
Yawei Wang ${ }^{1}$, Gang $\mathrm{Wu}^{2}$, Xueyan $\mathrm{Fu}^{2}$, Shaolin $\mathrm{Xu}^{2}$, Tianlong Wang ${ }^{1}$, Qi Zhang ${ }^{1}$ and Ye Yang ${ }^{1}$
}

\begin{abstract}
An increasing interest in liver cancer stemness arises owing to its aggressive behavior and poor prognosis. CD133, a widely known liver cancer stem cell marker, plays critical roles in the maintenance of liver cancer stemness. Thus, exploring the regulatory mechanism of CD133 expression is significant. In the present study, we proved the carcinogenesis roles of aquaporin 3 (AQP3) in hepatocellular carcinoma (HCC) and demonstrated that AQP3 promotes the stem cell-like properties of hepatoma cells by regulating CD133 expression. In addition, AQP3 promoted the stimulation and nuclear translocation of signal transducer and activator of transcription 3 (STAT3) with a subsequent increase in the level of CD133 promoter-acetylated histone H3. This phenomenon accelerated CD133 transcription. Next, whether AQP3 acted as an oncogenic gene in HCC and maintained the stemness of CD133+ hepatoma cells were elucidated; also, a novel mechanism underlying the AQP3/STAT3/CD133 pathway in HCC was deduced.
\end{abstract}

\section{Introduction}

In China, hepatocellular carcinoma (HCC) is the fourth most commonly diagnosed and the third most prevalent cancer leading to an estimated 422,100 deaths annually ${ }^{1}$. In recent decades, surgical resection is the first-line treatment for HCC; however, it is not applicable to the majority of the advanced $\mathrm{HCCs}^{2}$. The long-time survival is unsatisfactory due to the high rate of recurrence and metastasis. Liver cancer stem cells (LCSCs) are highly tumorigenic, metastatic, chemotherapy- and radiationresistant, and responsible for tumor relapse after ther$\mathrm{apy}^{3-5}$. Therefore, understanding the mechanism underlying the maintenance of LCSCs is crucial for future treatment strategies.

Aquaporin 3 (AQP3) belongs to aquaporins, a family of water channel proteins found in the plasma membranes of

Correspondence: Gang Wu (18660232387@163.com)

'Department of Geriatric Surgery, The First Affiliated Hospital of China Medical University, Shenyang, PR China

${ }^{2}$ Department of Hepatobiliary Surgery, The First Affiliated Hospital of China Medical University, Shenyang, PR China

Edited by A. Stephanou various cells ${ }^{6}$. Recent studies have shown that the aberrant expression of AQP3 contributes to the progression and metastasis of several malignant tumors ${ }^{7-10}$. Accumulating pieces of evidence support that AQP3 is related to the maintenance of stemness not only in normal stem cells but also in cancer stem cells $(\mathrm{CSCs})^{11-13}$. Another study in HCC cells indicated that AQP3 promotes cellular proliferation and invasion ${ }^{14}$. However, the AQP3 expression in HCC patients, the independent influence on prognosis, and the carcinogenic mechanism in $\mathrm{HCC}$ progression are yet to be clarified.

In the current study, we found AQP3 was highly expressed in HCC specimens and cell lines. The expression was related to TNM stage, metastasis, and prognosis in HCC. In vitro and in vivo experiments showed that the depletion of AQP3 suppresses the cellular proliferation and invasion. Further investigations revealed that AQP3 promotes the stem cell-like properties of hepatoma cells by regulating the expression of CD133. Interestingly, the janus kinase/signal transducer and activator of transcription 3 (JAK/STAT3) signaling pathway induces the expression of CD133 during liver carcinogenesis ${ }^{15}$,

\section{(c) The Author(s) 2019}

(c) Open Access This article is licensed under a Creative Commons Attribution 4.0 International License, which permits use, sharing, adaptation, distribution and reproduction cc) in any medium or format, as long as you give appropriate credit to the original author(s) and the source, provide a link to the Creative Commons license, and indicate if changes were made. The images or other third party material in this article are included in the article's Creative Commons license, unless indicated otherwise in a credit line to the material. If material is not included in the article's Creative Commons license and your intended use is not permitted by statutory regulation or exceeds the permitted use, you will need to obtain permission directly from the copyright holder. To view a copy of this license, visit http://creativecommons.org/licenses/by/4.0/. 
following which, we investigated whether AQP3 could affect the JAK/STAT3 signaling pathway. Results showed that AQP3 activated STAT3 by promoting STAT3 phosphorylation and inducing STAT3 nuclear translocation with a subsequent increase in the levels of acetylated histone $\mathrm{H} 3$ of the CD133 promoter. Taken together, we demonstrated that $A Q P 3$ functions as an oncogenic gene in HCC and maintains the stemness of CD133+ hepatoma cells. In addition, we identified a novel mechanism of the AQP3/STAT3/CD133 pathway in HCC.

\section{Materials and methods}

\section{Patient tissue samples and liver cancer cell lines}

HCC tissue slice samples were obtained from 120 patients diagnosed with HCC, who underwent a routine hepatic resection in the First Affiliated Hospital of China Medical University between January 2009 and January 2011. The inclusion criteria of all 120 patients were as follows: the tumor was completely resected without distant organ metastasis and the postoperative pathological diagnosis was HCC. The histological diagnosis and differentiation were evaluated independently by three pathologists using hematoxylin- and eosin-stained slides according to the WHO classification system ${ }^{16}$. None of the patients received preoperative radiotherapy or chemotherapy prior to surgical resection. The follow-up period for survival was 5 years. A total of 37 paired fresh specimens, including tumor tissues and the corresponding paired noncancerous parenchyma, were snap frozen in liquid nitrogen and stored at $-70^{\circ} \mathrm{C}$ immediately after resection. The inclusion criteria are the same as above. The project protocol was approved by the Institutional Ethics Committee of China Medical University prior to the initiation of the study. All patients provided informed consent before the study. Liver cancer cell lines Huh7, HCCLM3, SMMC7721, HepG2, Bel7402, PLC/PRF/5, and Hep3B and the normal liver cell line L02 were obtained from the Shanghai Cell Bank (Shanghai, China) and cultured in high-glucose Dulbecco's-modified Eagle medium supplemented with 10\% fetal bovine serum (FBS) and $1 \%$ penicillin/streptomycin in a humidified atmosphere containing $5 \% \mathrm{CO}_{2}$ at $37^{\circ} \mathrm{C}$.

\section{RNA preparation and quantitative real-time PCR}

Total RNA was extracted from $\sim 100 \mathrm{mg}$ of the 37 paired tissue samples and liver cancer cell lines using TRIzol (Invitrogen, USA) according to the manufacturer's instructions. The primers were designed and synthesized by Sangon Biotech Company (Shanghai, China) (Supporting file 1). The GAPDH gene was used as an endogenous control. The relative gene expression was assessed using qRT-PCR and expressed by $\Delta \mathrm{Ct}=\mathrm{Ct}$ gene- $\mathrm{Ct}$ reference; the fold-change in the gene expression was calculated using the $2^{-\Delta \Delta \mathrm{Ct}}$ method $^{17}$. Every tissue was assessed three times.

\section{Western blotting}

Total/cytoplasm/nucleus protein was extracted from tumor tissues, non-tumor adjacent tissues, or liver cancer cell lines using the Total/Cytoplasm/Nucleus Protein Extraction Kit (Solarbio, China). An equivalent of $50 \mu \mathrm{g}$ of the protein extract was resolved by sodium dodecyl sulfate polyacrylamide gel electrophoresis and electrotransferred to polyvinylidene difluoride membranes (Millipore, Billerica, MA, USA). The membrane was blocked for $2 \mathrm{~h}$ at room temperature using milk (5\%) was used to block membranes. Subsequently, the membranes were probed with primary antibodies, including rabbit polyclonal antibodies to AQP3 (1:2000, Abcam, USA), CD133 (1:1500, Abcam), JAK1 (1:1000, Abcam), pY-JAK1 (1:2000, Abcam), JAK2 (1:1500, Abcam), pY-JAK2 (1:1500, Abcam), STAT3 (1:2000, Abcam), pY $^{705}$-STAT3 (1:2000, Abcam), GAPDH mouse monoclonal antibody (1:2000, Abcam), Histone H3 (phospho S10) rabbit monoclonal antibody (1:500, Abcam) overnight at $4{ }^{\circ} \mathrm{C}$, followed by incubation with secondary antibodies for $2 \mathrm{~h}$ at room temperature. The immunoreactive bands were identified using an ECL system (Millipore, USA). Every tissue was evaluated three times using Western blotting.

\section{Immunofluorescence}

HCC cells were seeded in 12-well plates at moderate density and transfected as indicated above. The cells were fixed with $4 \%$ paraformaldehyde for $30 \mathrm{~min}$ and permeabilized by $1 \%$ Triton X-100 in phosphate-buffered saline (PBS) for $20 \mathrm{~min}$ at room temperature. After washing in PBS, the cells were incubated with $1 \%$ bovine serum albumin (BSA) for $30 \mathrm{~min}$. For immunofluorescence staining, the cells were incubated with AQP3 (1:1000, Abcam) or CD133 antibody (1:1000, Abcam). Then, goat anti-rabbit immunoglobulin G (1:2000, ProteinTech Group, USA) was used as a secondary antibody at $4{ }^{\circ} \mathrm{C}$ overnight. Finally, the cells were stained with $4^{\prime}, 6$-diamidino-2-phenylindole (Boster, China) to visualize the nuclei, and stained samples were imaged using a fluorescence microscope (Nikon eclipse, Japan). The immunofluorescence assay was conducted three times in each group.

\section{Immunohistochemistry (IHC)}

AQP3/CD133/CD44/CD90/EPCAM expression was analyzed in paraffin-embedded specimens obtained from 120 patients. Four- $\mu \mathrm{m}$-thick tissue sections were deparaffinized in xylene and dehydrated before antigen retrieval for 5 min using an autoclave. The endogenous peroxidase activity was blocked using hydrogen peroxide (0.3\%), and 
non-specific immunoglobulin binding sites were blocked by normal goat serum for $30 \mathrm{~min}$ at $37^{\circ} \mathrm{C}$. Tissue sections were incubated with anti-AQP3 (1:1000, Abcam), antiCD133 (1:200, Abcam), anti-CD44 (1:1000, Abcam), antiEPCAM (1:200, Abcam), and anti-CD90 (1:200, Abcam) overnight at $4{ }^{\circ} \mathrm{C}$. Then, the sections were incubated with biotinylated goat anti-rabbit IgG as a secondary antibody (Maixin Kit, China) for $1 \mathrm{~h}$ at room temperature, followed by incubation with streptavidin-biotin horseradish peroxidase-conjugated (Maixin Kit) for $30 \mathrm{~min}$ at room temperature. The peroxidase reaction was developed with 3 '-diaminobenzidine tetrahydrochloride (Maixin Kit). The expression levels of the proteins were scored semiquantitatively according to the percentage of positively stained cells combined with the staining intensity according to our previous study ${ }^{18}$. The specimens were assessed three times.

\section{Cell transfection}

The HCCLM3 and Huh7 cells were plated in 24-well plates until $60 \%$ confluence. Then, the cells were infected with lentivirus-AQP3-shRNAs or lentivirus-AQP3-NC (GenePharma, China) according to the multiplicity of infection values. After $12 \mathrm{~h}$, fresh medium was replaced. Subsequently, the expression of the GFP gene was observed under a fluorescence microscope. The effect of the shRNA-lentivirus on the expression of AQP3 was assessed by qRT-PCR. pcDNA-AQP3 was used to upregulated the AQP3 expression, and pcDNA3.1-NC was used as the control. Both plasmids were purchased from GenePharma.

\section{CCK8 and colony formation assay}

Approximately 2000 cells/well were plated in 96-well plates in media containing $10 \% \mathrm{FBS}$ at $24 \mathrm{~h}$ posttransfection. Then, $10 \mu \mathrm{l} \mathrm{CCK8}$ reagent (Solarbio, China) was added to each well and incubated for an additional $1 \mathrm{~h}$ at $37^{\circ} \mathrm{C}$, and the absorbance measured at $450 \mathrm{~nm}$.

After transfection, the cells were harvested in the logarithmic growth phase from the monolayer culture for the colony formation assay with $\sim 200$ cells/well in sixwell plates in media containing 10\% FBS. The colony formation was allowed for 2 weeks. Then, the cells were washed with $1 \mathrm{~mL}$ PBS, fixed, stained with $0.1 \%$ crystal violet solution for $20 \mathrm{~min}$, and finally washed three times with water. The fixed cell colonies were allowed to air dry, and the rate of clone formation was calculated. The CCK8 and colony formation assays were conducted three times in each group.

\section{Cell cycle analysis}

HCCLM3 and HUH7 cells in six-well plates were transfected with Lv-AQP3-shRNAs or Lv-AQP3-NC. After $48 \mathrm{~h}$ of transfection, the cells were seeded at a density of $5 \times 10^{5} /$ well, trypsinized, fixed with $70 \%$ ethanol at $4{ }^{\circ} \mathrm{C}$, and washed with PBS. A volume of $100 \mu \mathrm{L}$ RNase A was added, and the mixture was incubated in a $37^{\circ} \mathrm{C}$ water bath for $30 \mathrm{~min}$. An additional $400 \mu \mathrm{l}$ propidium iodide (PI; Sigma, USA) staining solution was added and incubated at $4{ }^{\circ} \mathrm{C}$ in the dark for $30 \mathrm{~min}$. Then fluorescence-activated cell sorting (FACS) Calibur (Becton Dickinson, USA) was used to detect and record the fluorescence upon excitation at $488 \mathrm{~nm}$. The cell cycle analysis was conducted three times in each group.

\section{Transwell assay}

After transfection, the cells were seeded on the synthetic basement membrane in the inset of a 24-well culture plate. In the invasion assay, polycarbonate filters coated with $50 \mu \mathrm{l}$ Matrigel (1:9, BD Bioscience, USA) were placed in the transwell chamber (Costar, USA). In the migration assay, no Matrigel was placed in the chambers. FBS was added to the lower chamber of the transwell as a chemoattractant. Then, the cells were incubated at $37^{\circ} \mathrm{C}$ to allow invasion through the Matrigel barrier for $24 \mathrm{~h}$. Subsequently, the filters were fixed and stained with $0.1 \%$ crystal violet solution. The non-invading cells were removed using a cotton swab, and invading cells on the underside of the filter were enumerated using an inverted microscope (Nikon MicrophotFX, Japan).

\section{Tumorigenicity experiments in nude mice}

A total of 30 male nude mice weighing 18-20 g, provided by Keygen Biotech (Nanjing, China), were bred under aseptic conditions and housed in the presence of constant humidity at $60-70 \%$ and room temperature of $18-20^{\circ} \mathrm{C}$. The animal maintenance, husbandry, and experimental procedures were performed in accordance with the rules of China Medical University for the Use of Experimental Animals and approved by the Medical Animal Care and Use Committee of China Medical University (Shenyang, China). Then, the mice were randomly assigned to three groups with HCCLM3 or HUH7 cells injected subcutaneously in the abdomen: Lv-AQP3NC, Lv-AQP3-shRNA1, Lv-AQP3-shRNA2. Subsequently, the animals were examined every 3 days, and killed at 21 days after the initial injection, followed by resection and weighing of the tumors.

\section{RT-PCR gene array}

Forty-eight hours after $A Q P 3$ knockdown in HCCM3 and HUH7 cells, total RNA was extracted from the cells using TRIzol (Invitrogen) and purified with the RNeasy_MinElute ${ }^{\mathrm{TM}}$ Cleanup Kit (Qiagen, Germany). Subsequently, the total RNA was reverse transcribed using Superscript III Reverse Transcriptase (Invitrogen), and the complementary DNA (cDNA) was amplified by PCR using 2_Super Array PCR Master Mix (Qiagen). Then, 
RT-PCR was performed on each sample using the Human Tumor Proliferation/Invasion RT2 Profiler ${ }^{\mathrm{TM}}$ PCR Array (SuperArray Bioscience, USA) in a Thermal Cycler Dice Real-Time System (Takara, Japan) according to the manufacturer's instructions. The expression data of the target genes were normalized to that of GAPDH using the $2^{-\Delta \Delta \mathrm{Ct}}$ method.

\section{FACS}

HCCLM3 and HUH7 cells were collected and washed with $1 \% \mathrm{BSA} /$ cold PBS two times by centrifugation at $1000 \mathrm{rpm}$ for $5 \mathrm{~min}$ at $4{ }^{\circ} \mathrm{C}$. CD133 rabbit polyclonal antibody (Abcam) was added to HCCLM3 or HUH7 cells and incubated for $15 \mathrm{~min}$ at room temperature in the dark. Then, the cells were washed with PBS, and goat antirabbit IgG-labeled FITC (Abcam, USA) was added to the cells for $15 \mathrm{~min}$ at room temperature, away from light. Subsequently, the cells were separated, and the proportion of CD133+ cells in HCCLM3 and HUH7 cells was estimated by FACS Calibur.

\section{Magnetic cell separation}

CD133 MicroBeads conjugated to monoclonal antihuman CD133 antibodies were purchased from Miltenyi Biotec (Germany). First, the CD133+ cells in HCCLM3 or HUH7 cells were magnetically labeled with CD133 MicroBeads according to the manufacturer's instructions. Then, the cell suspension was loaded onto the AutoMACS Pro Separator (Miltenyi Biotec). Consequently, the magnetically labeled CD133+ cells were retained and the unlabeled cells were excluded as the eluate. To increase the purity, the positively selected cell fraction containing the CD133+ cells was re-separated.

\section{Spheroid formation assay}

Sphere formation was performed in ultralow attachment plates (Corning, USA) containing medium supplemented with $2 \%$ B27 (Invitrogen), $20 \mathrm{ng} / \mathrm{ml}$ basic fibroblast growth factor (PeproTech, USA), and $20 \mathrm{ng} / \mathrm{ml}$ human recombinant epidermal growth factor (PeproTech, USA). CD133+ HCCLM3 and CD133+ HUH7 cells were plated at a density of 2 cells/ $\mu$ land cultured at $37{ }^{\circ} \mathrm{C}$ in $5 \% \mathrm{CO}_{2}$. After 14 days, the spheres $>50 \mu \mathrm{m}$ diameter were enumerated at $\times 40$ magnification under a microscope (Nikon).

\section{Side population assay}

The CD133+ HCCLM3 and HUH7 cell suspensions were labeled with Hoechst 33342 dye (Molecular Probes, USA) for side population analysis according to the standard protocol ${ }^{19}$. In brief, the cells were resuspended in OPTI-MEM (Gibco, USA) at a density of $10^{6} / \mathrm{ml}$. Hoechst 33342 dye was added at a final concentration of $5 \mu \mathrm{g} / \mathrm{ml}$ in the presence or absence of final concentration $50 \mu \mathrm{mol} / \mathrm{l}$ verapamil, and the cells were incubated at $37^{\circ} \mathrm{C}$ for
$90 \mathrm{~min}$ with intermittent shaking, followed by centrifugation at $1000 \mathrm{r} / \mathrm{min}$ at $4{ }^{\circ} \mathrm{C}$ for $10 \mathrm{mins}$. The cell pellet was suspended in chilled OPTI-MEM containing $2 \% \mathrm{FBS}$ and $10 \mathrm{mmol} / \mathrm{L}$ HEPES. In total, $1 \mu \mathrm{g} / \mathrm{ml} \mathrm{propi-}$ dium iodide was added to the cells to gate the viable cells during analysis on a FACS Calibur. The Hoechst 33342 dye was excited at $355 \mathrm{~nm}$.

\section{Chromatin immunoprecipitation analysis}

Chromatin immunoprecipitation (CHIP) was performed using the EpiQuik ${ }^{\mathrm{Tm}}$ Global Histone H3 Acetylation Assay Kit (Epigentek, USA) according to the manufacturer's instructions. In brief, cross-linked chromatin DNA was sonicated into 200-1000-bp fragments. This fragmented chromatin was immunoprecipitated using an anti-acetyl-histone H3. Normal mouse IgG was used as the negative control. qRT-PCR was performed to assess the expression of $\mathrm{CD} 133$ promoter.

\section{Statistical analysis}

The statistical analyses were performed using the SPSS 19.0 software. Parametric data are presented as mean \pm standard error of mean (SEM), and the differences between each group were analyzed using the Student's $t$ test. All the $p$ values reported were two-sided, and significance was defined as $p<0.05$.

\section{Results}

\section{AQP3 expression in HCC tissues and cell lines}

The expression of AQP3 mRNA was examined in 37 HCC fresh tissue samples using qRT-PCR. Compared with noncancerous tissues, high expression was detected in 27 cases (72.97\%) and low in eight cases (21.62\%); however, no difference was found among the noncancerous and cancer tissues in two cases (5.41\%) (Fig. 1a). Moreover, the mean expression levels of AQP3 mRNA were higher in HCC tissues as compared with the normal tissues $(p=$ 0.02, Fig. 1b). Among the 37 fresh tissue samples, 10 were selected randomly to assess the expression of AQP3 protein by western blot. The results showed seven cases with high expression, two with low, and one case with no difference in the expression (Fig. 1c). According to IHC in 120 HCC patients, AQP3 protein was found to be primarily expressed in the cytomembrane of the tumor cells (Fig. 1d). Furthermore, AQP3 was highly expressed in 70\% tumor samples $(84 / 120)$ and low or negatively expressed in the remaining 36 cases (30\%) (Table 1). Compared with the normal liver cell line L02, Huh7, and HCCLM3 cells showed maximal AQP3 expression among the seven liver cancer cell lines (Fig. 1e).

\section{Clinical significance of AQP3 expression in HCC}

The IHC results revealed an association between AQP3 expression and various clinicopathological factors in the 


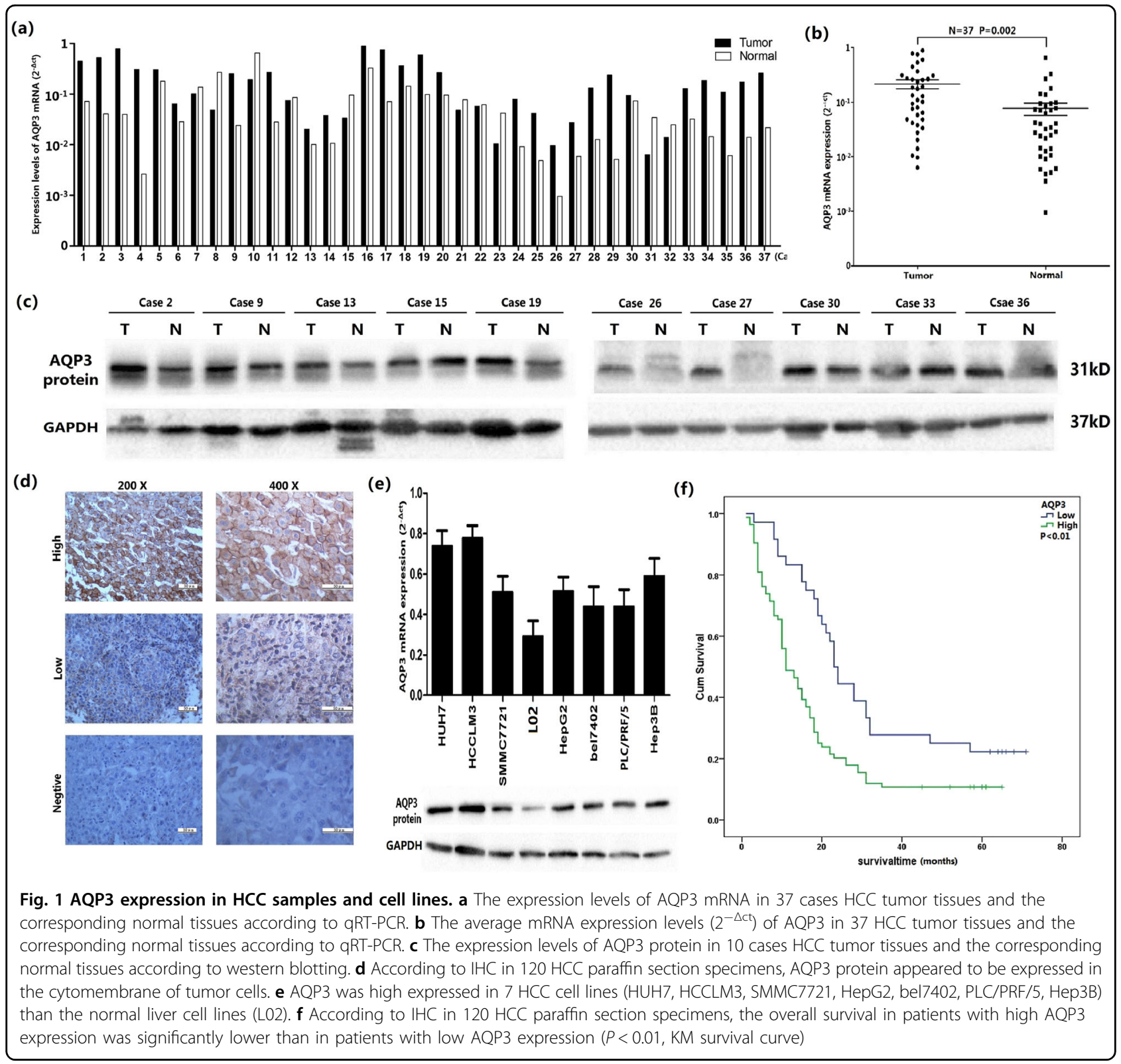

120 HCC patients. The AQP3 expression was significantly higher in HCC tissues with advanced TNM stage as compared with those with early TNM stage $(P=0.040)$, and the tissues with AQP3 overexpression exhibited marked tumor metastasis $(p=0.019$, Table 1$)$. Furthermore, patients with high AQP3 expression showed poorer prognosis. The overall survival was significantly lower in patients with high AQP3 expression than in those with low expression $(p<0.01$, Fig. 1f). In addition, multivariate analysis demonstrated that AQP3, TNM stage, and $\alpha-$ fetoprotein were significant prognostic factors for $\mathrm{HCC}$ patients (Table 2).

\section{Depletion of AQP3 inhibited HCC cellular proliferation and invasion}

Lv-AQP3-shRNAs were transfected into HCCLM3 and HUH7 cells with high AQP3 expression to investigate the roles of AQP3 on the malignant behavior of HCC cells. A significant reduction in the proliferation rate was observed at $48 \mathrm{~h}$ post transfection with Lv-AQP3-shRNAs as compared with that with Lv-AQP3-NC in both cell lines $(p<0.01$, Fig. 2a). Consistent with the results of the CCK8 assay, AQP3 knockdown led to a significant decrease in the number and size of foci as compared with the Lv-AQP3-NC group ( $p<0.01$, Fig. 2b). The effect of 
Table 1 Association between AQP3 expression according to immunohistochemistry and conventional clinicopathological parameters in 120 patients with HCC

\begin{tabular}{|c|c|c|c|c|}
\hline Characteristics & $\begin{array}{l}\text { Number of } \\
\text { patients }\end{array}$ & $\begin{array}{l}\text { AQP3 Low } \\
\text { expression }\end{array}$ & $\begin{array}{l}\text { AQP3 High } \\
\text { expression }\end{array}$ & $P$ \\
\hline & 120 & 36 & 84 & \\
\hline \multicolumn{5}{|l|}{ Age (years) } \\
\hline$\geq 50$ & 70 & 21 & 49 & \multirow[t]{2}{*}{0.582} \\
\hline$<50$ & 50 & 15 & 35 & \\
\hline \multicolumn{5}{|l|}{ Gender } \\
\hline Male & 80 & 25 & 55 & \multirow[t]{2}{*}{0.420} \\
\hline Female & 40 & 11 & 29 & \\
\hline \multicolumn{5}{|l|}{ Tumor size } \\
\hline$\geq 5 \mathrm{~cm}$ & 53 & 18 & 35 & \multirow[t]{2}{*}{0.260} \\
\hline$<5 \mathrm{~cm}$ & 67 & 18 & 49 & \\
\hline \multicolumn{5}{|l|}{ Metastasis } \\
\hline Yes & 52 & 10 & 42 & \multirow[t]{2}{*}{0.019} \\
\hline No & 68 & 26 & ; 42 & \\
\hline \multicolumn{5}{|l|}{ HBsAg status } \\
\hline Positive & 63 & 22 & 41 & \multirow[t]{2}{*}{0.150} \\
\hline Negative & 57 & 14 & 43 & \\
\hline \multicolumn{5}{|c|}{ Tumor differentiation } \\
\hline High & 14 & 3 & 11 & \multirow[t]{3}{*}{0.721} \\
\hline Moderate & 57 & 17 & 40 & \\
\hline Poor & 49 & 16 & 33 & \\
\hline \multicolumn{5}{|l|}{ Cirrhosis } \\
\hline Yes & 53 & 15 & 37 & \multirow[t]{2}{*}{0.485} \\
\hline No & 67 & 21 & 47 & \\
\hline \multicolumn{5}{|l|}{ Serum AFP } \\
\hline$<200 \mathrm{ng} / \mathrm{dl}$ & 39 & 13 & 26 & \multirow[t]{2}{*}{0.364} \\
\hline$\geq 200 \mathrm{ng} / \mathrm{dl}$ & 81 & 23 & 58 & \\
\hline \multicolumn{5}{|l|}{ Recurrence } \\
\hline Yes & 83 & 26 & 57 & \multirow[t]{2}{*}{0.402} \\
\hline No & 37 & 10 & 27 & \\
\hline \multicolumn{5}{|l|}{ TNM stage } \\
\hline$I+\|$ & 55 & 23 & 22 & \multirow[t]{2}{*}{0.040} \\
\hline$I I I+I V$ & 65 & 13 & 52 & \\
\hline
\end{tabular}

Bold fonts indicate statistically significant

AQP3 on the cell cycle was assessed using flow cytometry analysis. The results showed that the depletion of AQP3 increased the percentage of cells in G1 phase and decreased that in the $\mathrm{S}$ phase in both cell lines $(p<0.01$, Fig. 2c). In vivo, a xenograft nude mice model was used to test the influence of AQP3 on tumorigenicity. After 21 days post cell injection, lower tumor weight and volume were observed in Lv-AQP3-shRNAs HCCLM3 and HUH7 groups as compared with the Lv-AQP3-NC groups (Fig. 3). The cell invasion and migration assays demonstrated that HCCLM3 cells transfected with LvAQP3-shRNA1 or Lv-AQP3-shRNA2 showed lessintense invasive and migratory capacities as compared with the control group $(p<0.01$, Fig. $2 \mathrm{~d})$. The same phenomenon was also observed in HUH7 cells $(p<0.01$, Fig. 2e).

\section{AQP3 expression was positively correlated with CD133}

In order to explore the mechanism of AQP3 carcinogenesis, the mRNA expression profile of Lv-AQP3shRNA1-transfected HCCLM3 and HUH7 cells was compared with that of Lv-AQP3-NC transfected cells using a Human Tumor Proliferation/Invasion RT2 Profiler $^{\text {TM }}$ PCR Array containing 86 cell proliferation/metastasis-related genes (Supporting files 1, 2). Four LCSC markers (CD133, CD44, CD90, and EPCAM) were downregulated after AQP3 knockdown in both two cell lines (Fig. 4a). Next, we examined the correlation between $\mathrm{AQP} 3$ and the four LCSC markers in HCC specimens by qRT-PCR and IHC. The results indicated that the mRNA levels of AQP3 and CD133 were positively correlated in 37 HCC fresh tissue samples (Fig. 4b). Furthermore, a positive correlation was detected between the protein levels of AQP3 and CD133 according to IHC in $120 \mathrm{HCC}$ patients. The IHC data showed that patients with high expression of AQP3 exhibited higher levels of CD133 as compared with those with low expression ( $p=0.007$, Fig. $4 \mathrm{~g}, \mathrm{~h})$. However, no significant correlation was observed between AQP3 and the other three LCSC markers (Fig. 4c-g).

\section{AQP3 regulated CD133 expression in HCC}

FACS was used to evaluate the proportion of CD133+ cells in HCCLM3 and HUH7 cell lines: $5.5 \pm 0.7 \%$ and $7.1 \pm 0.9 \%$, respectively (Fig. $5 \mathrm{a}$ ). Then, we transfected $\mathrm{Lv}$ AQP3-shRNAs into HCCLM3 and HUH7 cells. Compared with the negative control and Lv-AQP3-NC groups, the CD133+ cell population in Lv-AQP3-shRNAs groups was significantly decreased $(p<0.01$, Fig. $5 \mathrm{a})$.

The CD133+ subpopulation was purified by magnetic cell separation (MACS). Subsequently, the sorted CD133 + cells were cultured for $48 \mathrm{~h}$ (Fig. $5 \mathrm{~b}$ ) and transfected with Lv-AQP3-NC or Lv-AQP3-shRNAs, followed by the evaluation of the expression of CD133 mRNA and protein by qRT-PCR and Western blotting. The results showed that AQP3 knockdown suppressed the expression of CD133 mRNA and protein in CD133+ HCCLM3 and HUH7 cells (Fig. 5c, d). Double immunofluorescent staining assays were used to test the colocalization of AQP3 and CD133. Results showed AQP3 and CD133 
Table 2 COX regeression regression analysis on the relationship of clinicopathologic characteristics and prognosis

\begin{tabular}{|c|c|c|c|c|c|c|}
\hline \multirow[t]{2}{*}{ Characteristics } & \multicolumn{3}{|c|}{ Univariate } & \multicolumn{3}{|c|}{ Multivariate } \\
\hline & HR & $\mathrm{Cl}(95 \%)$ & $P$ & HR & $\mathrm{Cl}(95 \%)$ & $P$ \\
\hline Age & 0.913 & $0.621-1.357$ & 0.648 & & & \\
\hline Gender & 1.302 & $0.867-1.952$ & 0.203 & & & \\
\hline TNM stage & 1.492 & $1.220-1.826$ & $<0.001$ & 1.418 & $1.150-1.748$ & 0.001 \\
\hline Tumor differentiation & 1.017 & $0.761-1.358$ & 0.911 & & & \\
\hline Metastasis & 1.612 & $1.092-2.379$ & 0.016 & 1.163 & $0.769-1.758$ & 0.474 \\
\hline Recurrence & 1.320 & $0.862-2.021$ & 0.201 & & & \\
\hline Tumor size & 0.822 & $0.556-1.214$ & 0.324 & & & \\
\hline Serum AFP & 1.843 & $1.196-2.840$ & 0.006 & 1.803 & $1.157-2.810$ & 0.009 \\
\hline Cirrhosis & 1.026 & $0.695-1.514$ & 0.898 & & & \\
\hline HBsAg status & 0.871 & $0.592-1.283$ & 0.486 & & & \\
\hline AQP3 & 2.027 & $1.307-3.145$ & 0.002 & 1.955 & $1.256-3.044$ & 0.003 \\
\hline
\end{tabular}

Bold fonts indicate statistically significant

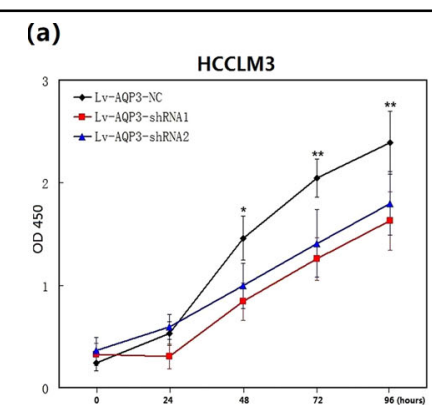

(b)

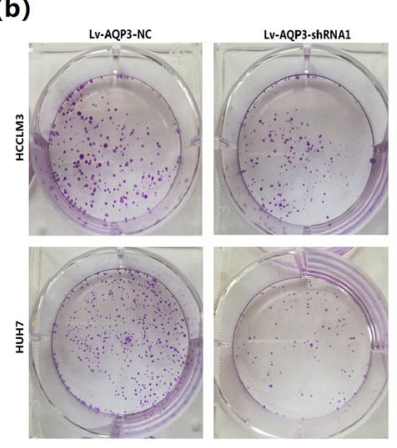

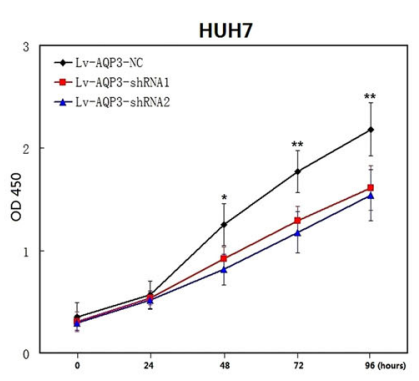

(c)

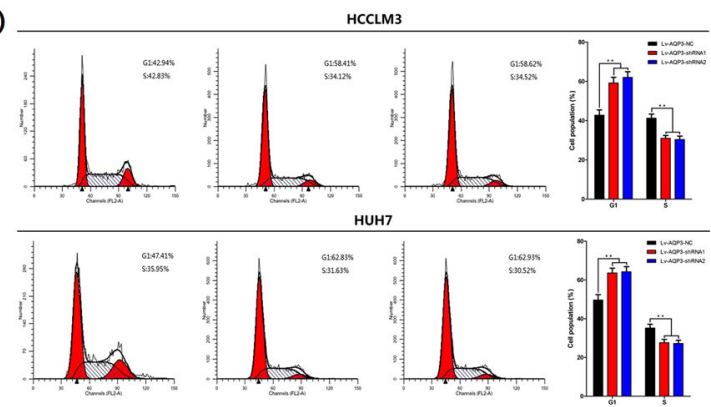

(d)
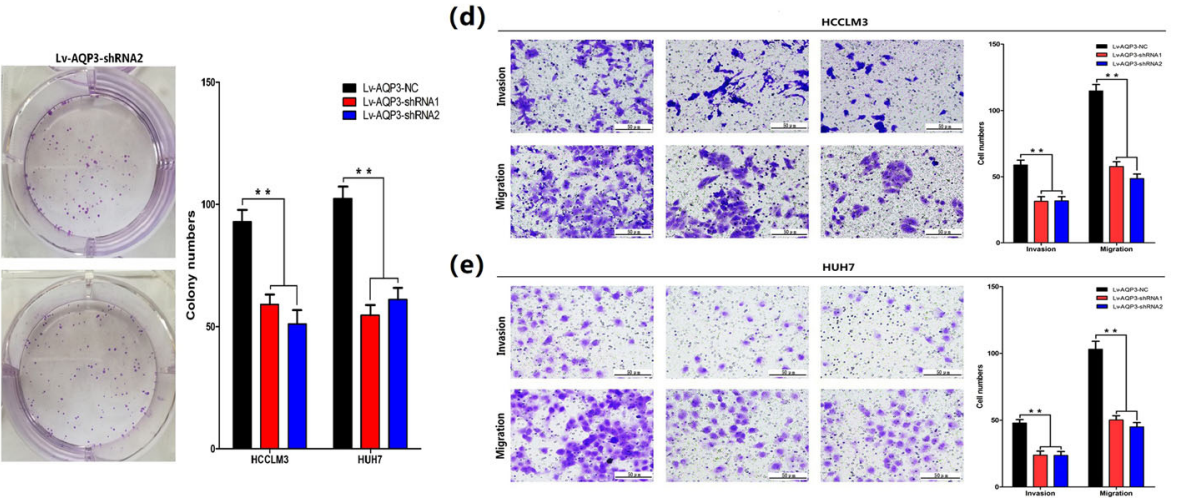

Fig. 2 Depletion of AQP3 inhibited HCC celluar proliferration. a According to CCK8 assays, significant decreased proliferation rate was observed $48 \mathrm{~h}$ post transfection with Lv-AQP3-shRNAs compared with LV-AQP3-NC in HCCLM3 and HUH7 cells $\left({ }^{*} P<0.05,{ }^{*} P<0.01\right)$. b Clonogenic assays were performed with HCCLM3 and HUH7 cells. Lower number of colonies were found in cells treated with LV-AQP3-shRNAs compared with Lv-AQP3-NC treated cells ( $\left.{ }^{* *} P<0.01\right)$. c The effect of AQP3 on the cell cycle was tested using flow cytometry analysis. In HCCLM3 and HUH7 cells, AQP3 depletion cells showed an increase in the number of cells in G1 phase and an decrease in the number of cells in S phase when compared with the LV-AQP3-NC groups ( $\left.{ }^{*} P<0.01\right)$. d e Transwell assays showed lower invasion and migration abilities were observed in HCCLM3 and HUH7 cells transfected with Lv-AQP3-shRNAs compared with Lv-AQP3-NC $\left({ }^{* *} P<0.01\right)$ 


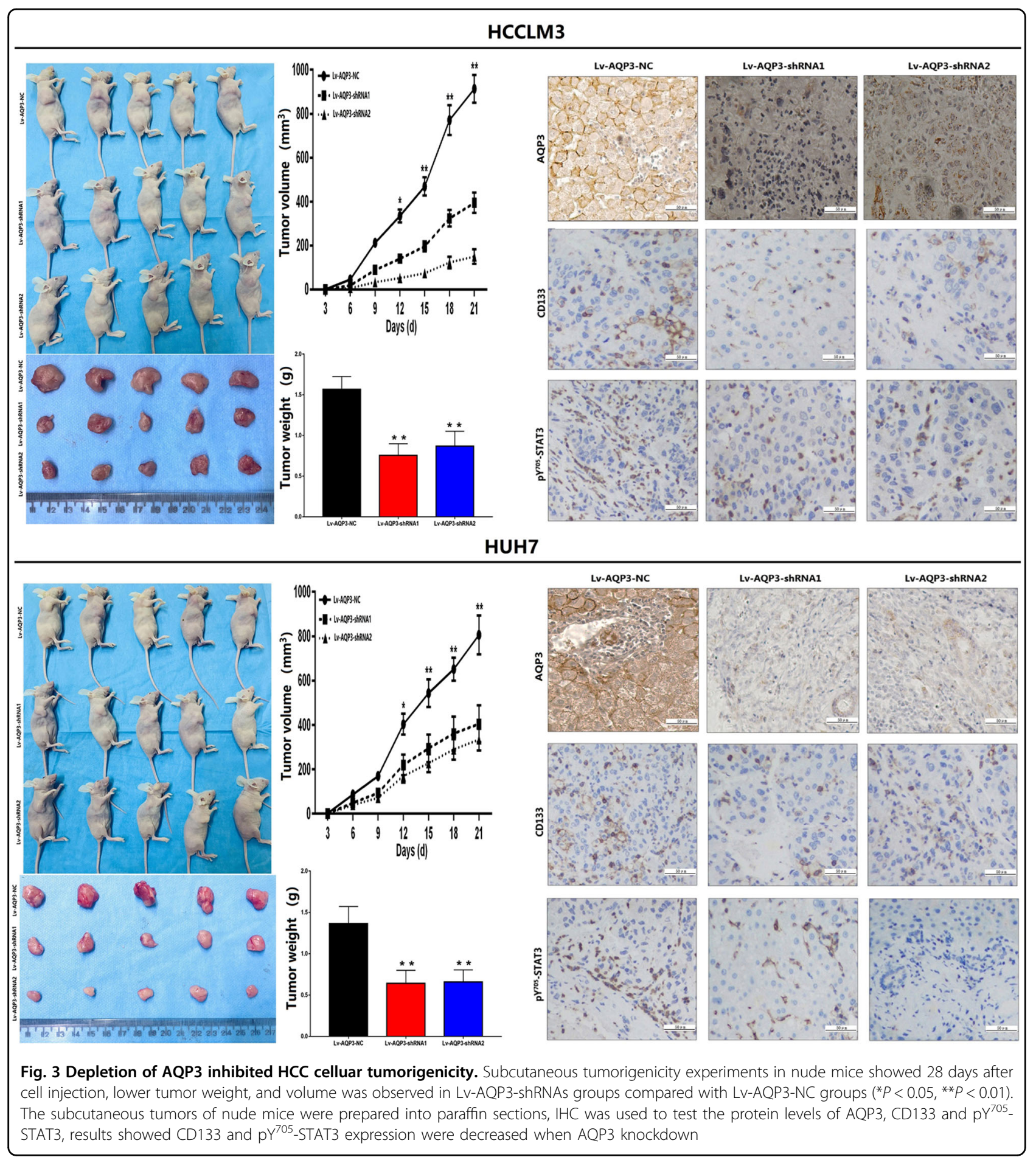

were co-expressed and colocalized in CD133+ HCC cells. And when we knocked down AQP3 expression, the CD133 expression was also downregulated (Fig. 6).

\section{AQP3 maintained the stemness of CD133+ HCC cells}

The spheroid formation assay was performed to assess the effect of AQP3 on the self-renewal capacity in CD133
+ HCC cells. As shown in Fig. 7a, the number of spheroids formed decreased significantly when AQP3 expression in CD133+ HCCLM3 and HUH7 cells was downregulated due to Lv-AQP3-shRNAs $(p<0.01)$. The Hoechst staining and flow cytometry sorting were employed to test the side population proportion in CD133 + HCCLM3 and HUH7 cells. The results showed that 


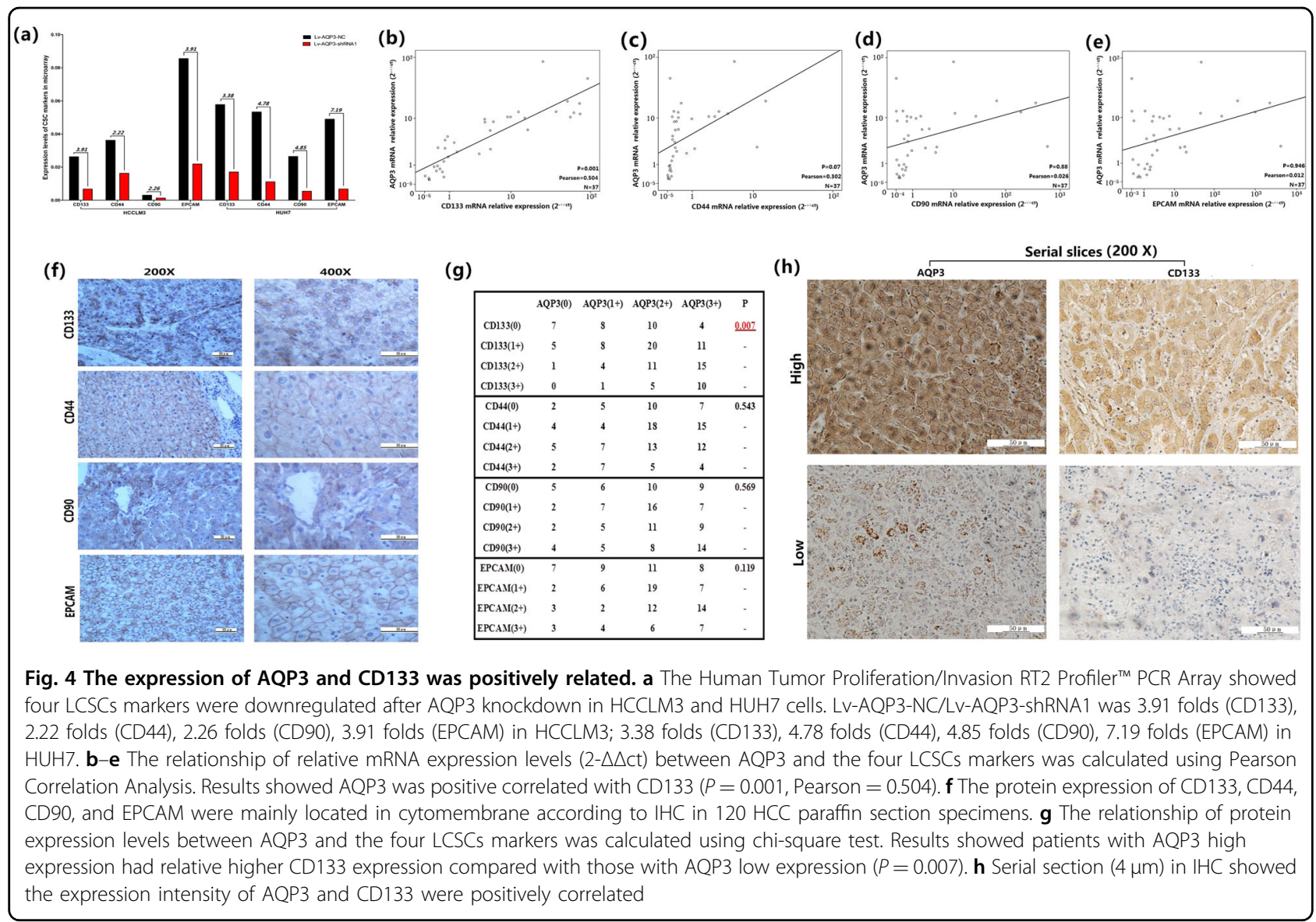

AQP3 depletion reduced the SP proportion in CD133+ HCCLM3 and HUH7 cells as compared with the control groups $(p<0.01$, Fig. $7 b, c)$. Then, we upregulated AQP3 expression by pcDNA-AQP3 in CD133+ HCC cells. The number of spheroids and the side population proportion were both increased as compared with the control groups $(P<0.05$, Fig. $7 \mathrm{a}-\mathrm{c})$.

\section{AQP3 regulated the activation of STAT3}

Several studies have shown that the JAK/ STAT3 signaling pathway contributed to the induction and maintenance of CSCs via the transcriptional regulation of $\mathrm{CD} 133^{20-22}$. In order to explore the mechanism underlying the AQP3-regulated CD133 expression, we analyzed the influence of AQP3 on JAK/STAT3 signaling pathway. The results showed that AQP3 did not affect the protein expression levels of JAK1, activated JAK1 (pYJAK1), JAK2, activated JAK2 (pY-JAK2), and STAT3 (Fig. 5e). Furthermore, AQP3 knockdown suppressed the expression of activated STAT3 (pY ${ }^{705}$-STAT3) (Fig. 5e) and blocked its nuclear translocation (Fig. 5f). Then, pcDNA-AQP3 was transfected to upregulate the expression of AQP3 in HCC cells. In addition, we found that
$\mathrm{pY}^{705}$-STAT3 had higher expression levels in the pcDNAAQP3 group than in the pcDNA-NC groups. Also, AG490 (STAT3 activation inhibitor, MedchemExpress, USA) counteracted the active effect of AQP3 on STAT3 (Fig. $5 \mathrm{~g})$. In vivo experiment, we tested the CD133 and $\mathrm{pY}^{705}$. STAT3 expression levels in sections of nude mice subcutaneous tumors by IHC, results showed CD133 and $\mathrm{pY}^{705}$-STAT3 protein levels were decreased when AQP3 knockdown (Fig. 3), which was consistent with the variations in volume of subcutaneous tumors.

\section{AQP3 promoted CD133 transcription depending on STAT3 activation}

Next, we upregulated the expression of AQP3 via pcDNA-AQP3 transfection to investigate the effect of AQP3 overexpression on CD133+ cell population ratio, CD133 expression, and CD133 promoter (p-CD133)acetylated histone H3 levels in HCCLM3 and HUH7 cells. Moreover, we tested whether AG-490 inhibited the AQP3-mediated CD133 upregulation upon STAT3 activation. HCCLM3 and HUH7 cells were divided into six groups: negative control, pcDNA-NC, pcDNA-AQP3, negative control + AG-490, pcDNA-NC + AG-490, and 


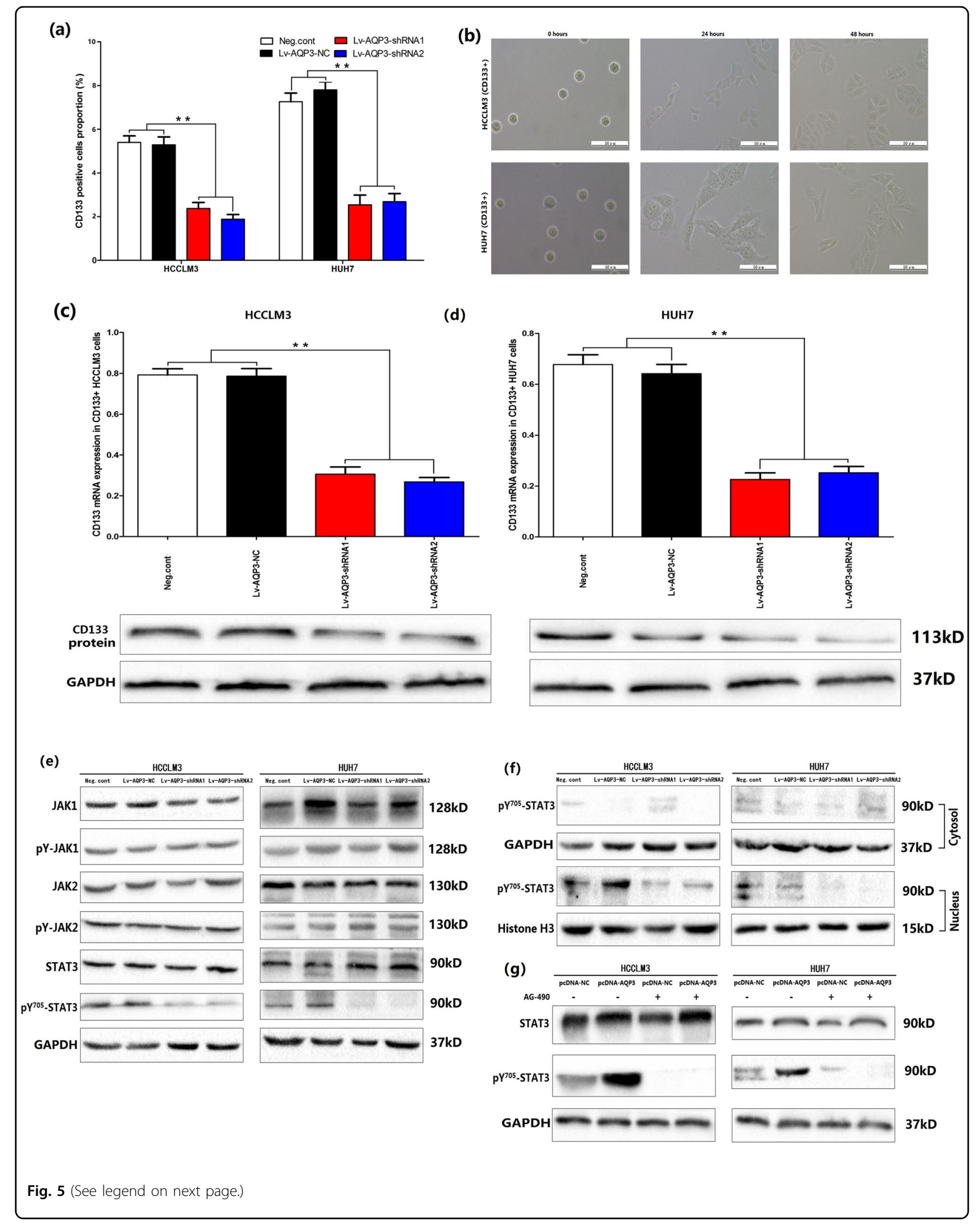


(see figure on previous page)

Fig. 5 AQP3 regulated CD133 and pY ${ }^{705}$-STAT3 expression. a FACS was used to test the CD133 positive cells proportion in HCCLM3 and HUH7 cell lines. Results showed AQP3 knockdown could reduce CD133+ cells proportion (**P<0.01). b The purification of CD133+ subpopulation in HCCLM3 and HUH7 cells was performed by MACS. The sorted CD133+ cells were displayed under electron microscope and cultured for $48 \mathrm{~h}$ into logarithmic phase to transfected Lv-AQP3-shRNAs or Lv-AQP3-NC. c, d qRT-PCR, western blotting showed AQP3 knockdown significantly inhibited CD133 mRNA and protein expression in CD133+ HCCLM3 and HUH7 cells (**P $<0.01)$. e The key protein levels in JAK/STAT3 signaling pathway was tested by western blotting in HCCLM3 and HUH7 cells. Results showed AQP3 konckdown could depress the activating STAT3 (pY ${ }^{705}$-STAT3) expression. $\mathbf{f}$ In HCC cells, PY ${ }^{705}$-STAT3 was rarely expressed in the cytoplasm and mainly expressed in the nucleus. AQP3 konckdown could significantly decrease $\mathrm{pY}^{705}$-STAT3 nuclear translocation; $\mathbf{g}$ PCDNA-AQP3 was used to upregulated AQP3 expression. We found AQP3 overexpression had no influence on STAT3 expression, but could promote STAT3 activation. AG-490 treatment could inhibit STAT3 activation and eliminate the influence of $\mathrm{AQP3}$ overexpression

pcDNA-AQP3 + AG-490. First, we assessed the CD133+ cell population ratio using FACS methods and found that the overexpression of AQP3 increased the ratio of CD133 + cell population in HCCLM3 and HUH7 cells $(p<0.01$, Fig. 8a). AG-490 decreased the ratio $(P<0.01)$ and eliminated the influence of AQP3 overexpression $(p<$ 0.01, Fig. 8a). Second, we tested the CD133 mRNA and protein expression using qRT-PCR and western blotting, respectively. Furthermore, we found that the overexpression of AQP3 upregulated the expression of CD133 ( $p<0.01$, Fig. 8b). AG-490 downregulated the expression of CD133 $(p<0.01)$ and eliminated the influence of AQP3 overexpression $(p<0.01$, Fig. $8 \mathrm{~b})$. Finally, we detected the CD133 promoter-acetylated histone H3 levels using ChIP analysis. The results showed AQP3 knockdown decreased the CD133 promoter-acetylated histone H3 levels $(p<0.01$, Fig. 8e, f). Moreover, the overexpression of AQP3 increased the CD133 promoter-acetylated histone H3 levels $(p<0.01$, Fig. 8g, h), whereas AG-490 decreased the levels $(p<0.01)$ and eliminated the influence of AQP3 overexpression $(p<0.01$, Fig. $8 \mathrm{~g}, \mathrm{~h})$. Furthermore, we tested the influence of AG-490 on the stemness of CD133 + HCC cells. Results showed AG-490 could not only decrease the number of spheroids and the side population proportion in CD133+ HCC cells ( $p<0.01$, Fig. $8 \mathrm{c}$, d), but also eliminated the influence of AQP3 overexpression on the stemness of CD133+ HCC cells $(p<0.01$, Fig. 8c, d).

\section{Discussion/conclusion}

CD133, a widely known LCSC marker, has been proved to promote HCC proliferation and invasion ${ }^{23-25}$. CD133 + HCC cells possess high capacity of tumorigenicity and strong ability of self-renewal ${ }^{26,27}$. Accumulating evidence showed that $\mathrm{CD} 133$ could be used to predict and diagnose the development of $\mathrm{HCC}^{28-30}$. Thus, clarifying the molecular mechanism underlying the abnormal expression of CD133 during tumor progression in $\mathrm{HCC}$ is significant.

AQP3 is expressed in various cancer cells derived from diverse types of cancer tissues from stomach, colon, and lung $^{31-33}$. In breast cancer, AQP3-facilitated cellular uptake of hydrogen peroxide promotes cell migration by regulating the Akt pathway ${ }^{34}$. Recent studies indicated a close correlation between AQP3 and stemness maintenance not only in normal stem cells but also in CSCs ${ }^{11-13}$.

Nevertheless, the significant role and the molecular mechanism of AQP3 in HCC progression have rarely been reported. Only one study showed that the combined overexpression of AQP3 and AQP5 in HCC might be strongly related to tumor progression and prognosis in patients with $\mathrm{HCC}^{35}$. However, the independent effect of AQP3 on malignant behavior of hepatoma cells and the related mechanisms are yet unknown.

In current study, we found that AQP3 was highly expressed in HCC and exerted carcinogenic roles and also delved into the mechanism underlying carcinogenesis. Thus, qRT-PCR gene array was used to screen the abnormal signal markers of AQP3 overexpression in HCC cell lines. The results showed that AQP3 expression was correlated with four LCSC markers (CD133, CD44, CD90, and EPCAM). Further experiments substantiated a positive correlation between AQP3 and CD133 expression in HCC samples, while the similar phenomenon was not observed for the other three markers. Follow-up experiments revealed that AQP3 not only regulated the CD133 + cell proportion in HCCLM3 or HUH7 cells but also stimulated the CD133 expression and promoted the stem cell-like properties in CD133 + HCCLM3 and HUH7 cells. Thus, the intrinsic molecular mechanism of AQP3regulated CD133 expression demands further exploration.

Recent studies demonstrated that CD133 is encoded by an inducible gene, and its expression at the transcriptional level is directly regulated by interleukin-6 (IL-6)-mediated activation of STAT3 ${ }^{15,36}$. The binding of IL- 6 to its receptor gp130 causes receptor dimerization and subsequent activation of the associated JAKs. These activated JAKs, phosphorylate the receptor which now serves as the docking site for STAT3 also phosphorylated by JAKs ${ }^{37-39}$. The activated STAT3 translocates into the nucleus and directly binds to the promoter of CD133 leading to increased histone acetylation and subsequent transcription of CD133 ${ }^{15,36}$.

In order to explore the mechanism of AQP3-regulated CD133 expression, we analyzed the influence of AQP3 on 


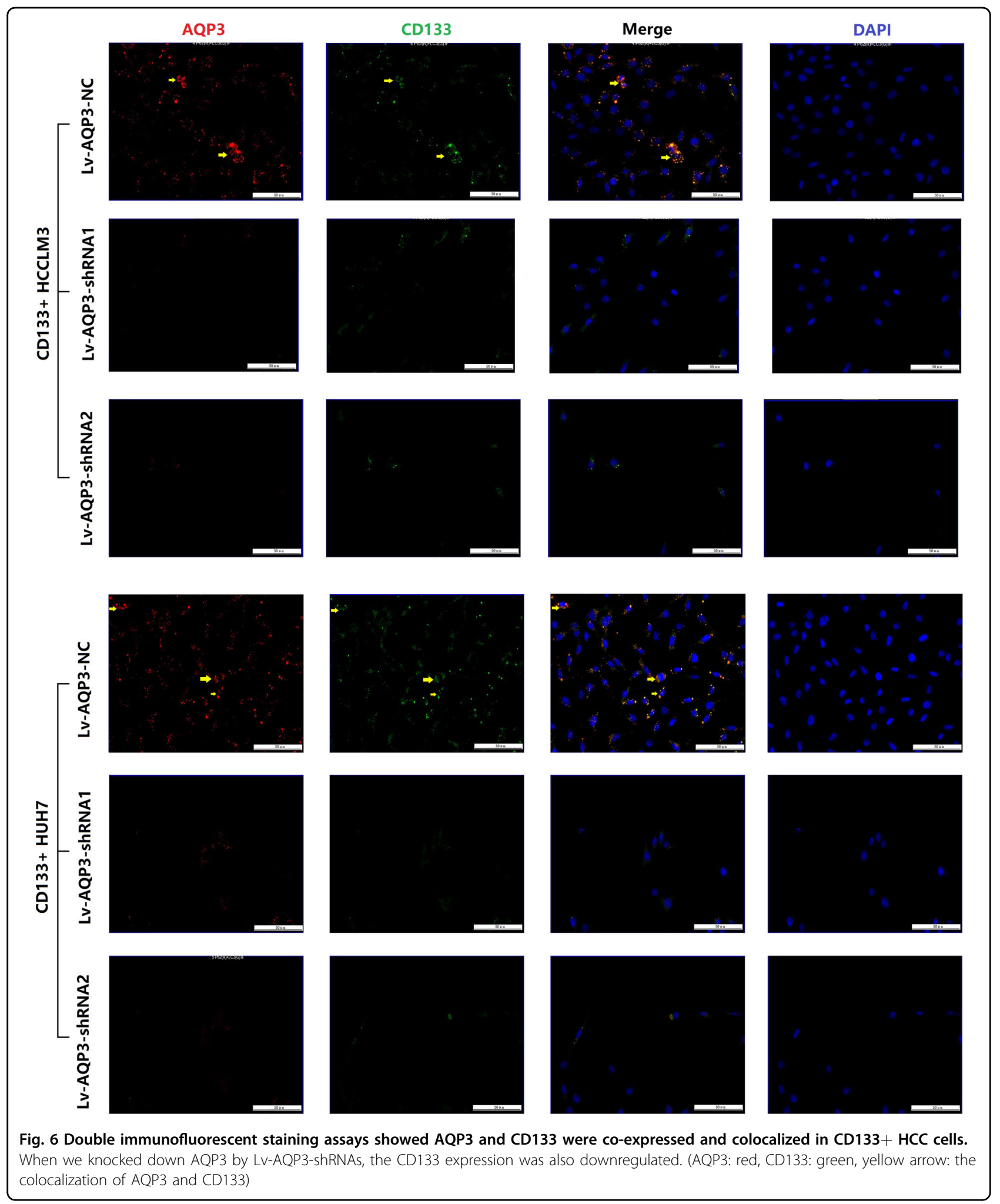

JAK/STAT3 signaling pathway. The results showed that AQP3 knockdown could suppress the activated STAT3 $\left(\mathrm{pY}^{705}\right.$-STAT3) expression and blocked the $\mathrm{pY}^{705}$-STAT3 nuclear translocation. The AQP3 overexpression promoted STAT3 activation, and the STAT3 activation inhibitor, AG-490, counteracted the active effect of AQP3 


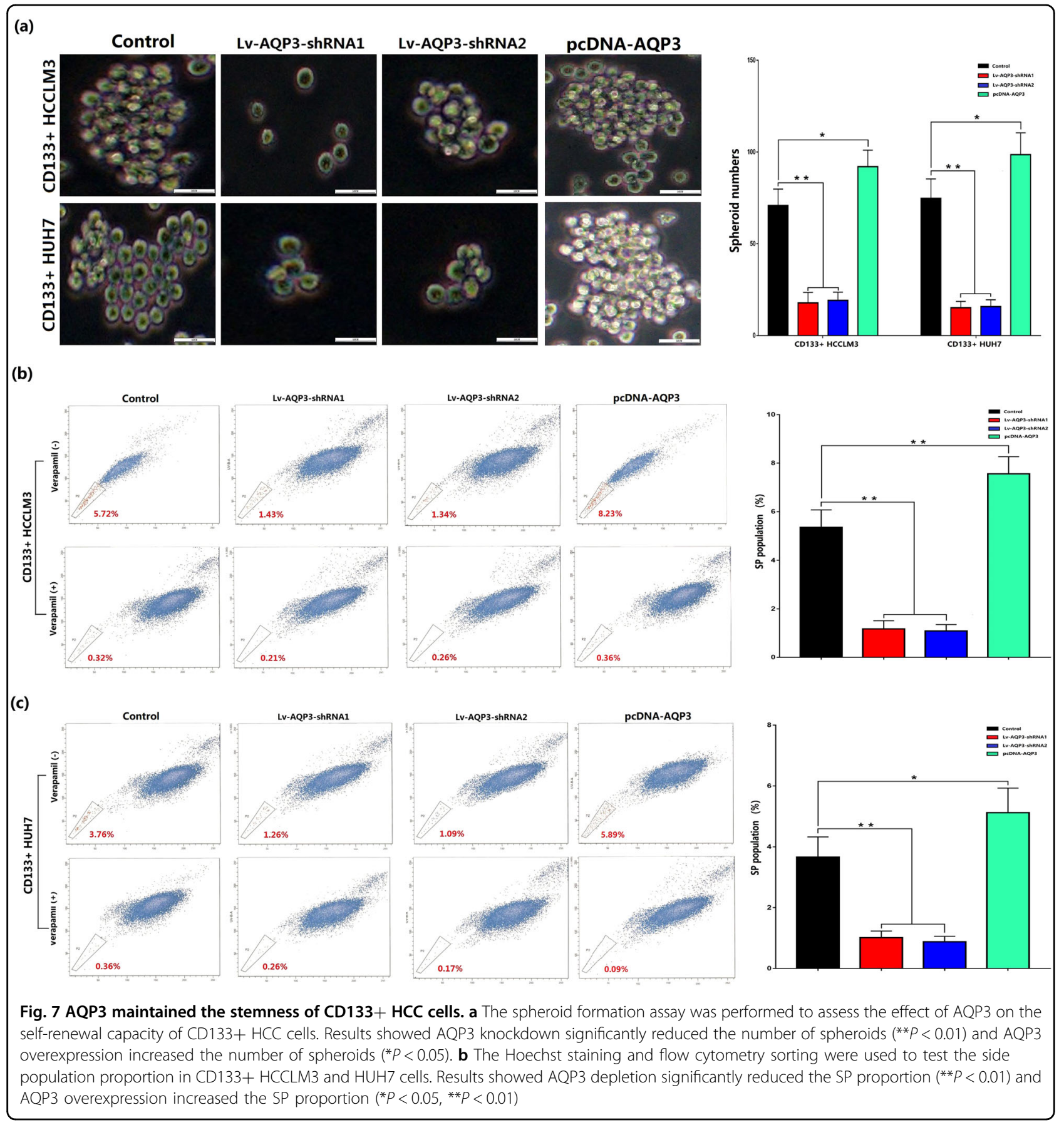

on STAT3. Next, we investigated the effect of AQP3 overexpression on CD133 + cell population ratio, CD133 expression, and CD133 promoter (p-CD133)-acetylated histone H3 levels in HCCLM3 and HUH7 cells. The results showed the AQP3 overexpression increased CD133 + cell proportion, CD133 expression, and pCD133-acetylated histone H3 levels. Then, we used AG490 to validate the effect of AQP3-mediated CD133 upregulation on STAT3 activation. Thus, we concluded that AG-490 not only decreased the CD133 + cell population ratio, CD133 expression, and p-CD133-acetylated histone $\mathrm{H} 3$ levels in $\mathrm{HCC}$ cells but also eliminated the influence of AQP3 overexpression. Therefore, it could be speculated that AQP3 activated STAT3 promotes the transcription of CD133.

In summary, we clarified the carcinogenic molecular mechanism of AQP3/STAT3/CD133 signaling pathway in HCC by four pieces of experimental evidence: (a) AQP3 


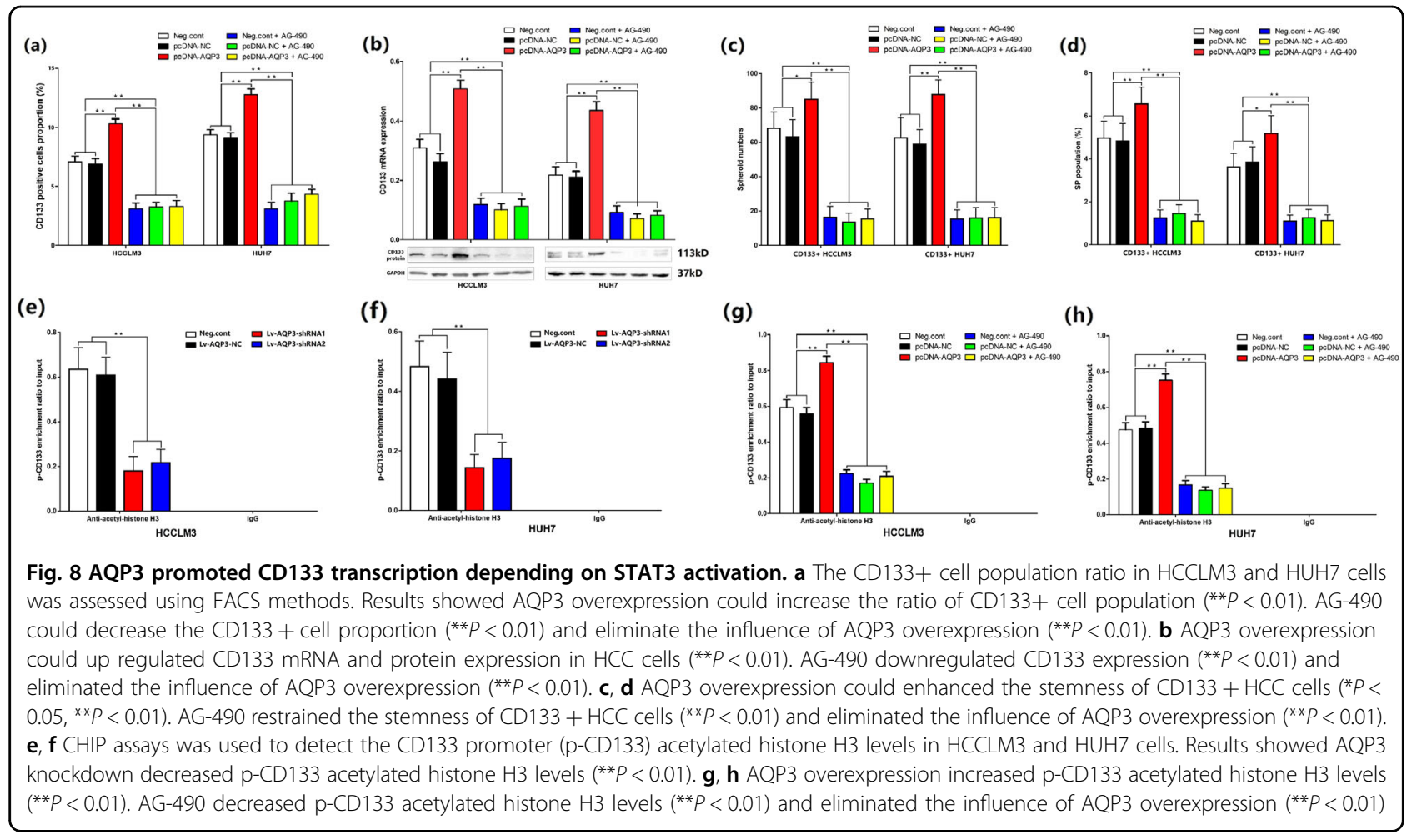

was overexpressed and executed oncogenic roles in HCC; (b) AQP3 upregulated the expression of CD133 and maintained the stemness of CD133+ HCC cells; (c) AQP3 accelerated the activation of STAT3; (d) AG-490 (STAT3 activation inhibitor) eliminated the promotion effects of AQP3 on CD133 transcription.

These findings indicated that AQP3 plays a major role in hepatic carcinogenesis and is closely related to the characteristics of LCSCs. This might provide a novel target or method for the detection and treatment of $\mathrm{HCC}$ in the future.

\section{Acknowledgements}

This work was supported by The Core Laboratory of The First Affiliated Hospital of China Medicai Univercity. This work was supported by a grant from Population and Health Research Projects from Shenyang Science and Technology Bureau (No. F15-139-9-25). This work was supported by a grant from Natural Science Foundation of Liaoning Province (No. 201520529). This work was supported by a grant from Youth Project of China Medical University (No. QGZ2018038).

\section{Author contributions}

Gang Wu designed the experiments. Yawei Wang and Xueyan Fu conducted all experiments. Yawei Wang wrote the manuscript. Shaolin $\mathrm{Xu}$, Wanchuan Zhang, Tianlong Wang, Qi Zhang, Ye Yang contributed with data analysis and discussed the results. All authors read and approved the final manuscript.

\section{Data availability}

All data generated or analysed during this study were included in this published article and its Supplementary Information Files.

\section{Conflict of interest}

The authors declare that they have no conflict of interest.

\section{Consent for publication}

All patients in current study had consent for publication and signed the informed consent.

\section{Ethics approval and consent to participate}

All patients in current study have read and signed an informed consent. The project protocol was approved by the Institutional Ethics Committee of China Medical University prior to the initiation of the study. In current study, the animal maintenance, husbandry and experimental procedures were performed in accordance with the rules of China Medical University for the Use of Experimental Animals and approved by the Medical Animal Care and Use Committee of China Medical University (Shenyang, China).

\section{Publisher's note}

Springer Nature remains neutral with regard to jurisdictional claims in published maps and institutional affiliations.

Supplementary Information accompanies this paper at (https://doi.org/ 10.1038/s41419-019-1712-0).

Received: 11 March 2019 Revised: 28 May 2019 Accepted: 29 May 2019 Published online: 13 June 2019

\section{References}

1. Chen, W. et al. Cancer statistics in China, 2015. CA Cancer J. Clin. 66, 115-132 (2016).

2. Singal, A. G. et al. Racial, social, and clinical determinants of hepatocellular carcinoma surveillance. Am. J. Med. 128, e1-e7 (2015). 90.

3. Nio, K., Yamashita, T. \& Kaneko, S. The evolving concept of liver cancer stem cells. Mol. Cancer. 16, 4 (2017).

4. Cheng, Z., Li, X. \& Ding, J. Characteristics of liver cancer stem cells and clinical correlations. Cancer Lett. 379, 230-238 (2016). 
5. Oikawa, T. Cancer Stem cells and their cellular origins in primary liver and biliary tract cancers. Hepatology. 64, 645-651 (2016).

6. Verkman, A. S. Aquaporins in clinical medicine. Annu. Rev. Med. 63, 303-316 (2012).

7. Wang, J. et al. c-Met upregulates aquaporin 3 expression in human gastric carcinoma cells via the ERK signalling pathway. Cancer Lett. 319, 109-117 (2012).

8. Wang, $X$. et al. AQP3 small interfering RNA and PLD2 small interfering RNA inhibit the proliferation and promote the apoptosis of squamous cell carcinoma. Mol. Med. Rep. 16, 1964-1972 (2017).

9. Huang, X., Huang, L. \& Shao, M. Aquaporin 3 facilitates tumor growth in pancreatic cancer by modulating mTOR signaling. Biochem. Biophys. Res. Commun. 486, 1097-1102 (2017).

10. Xiong, G. et al. RNA interference influenced the proliferation and invasion of XWLC-05 lung cancer cells through inhibiting aquaporin 3. Biochem. Biophys. Res. Commun. 485, 627-634 (2017).

11. Graziano, A. C. E., Avola, R., Pannuzzo, G. \& Cardile, V. Aquaporin1 and 3 modification as a result of chondrogenic differentiation of human mesenchymal stem cell. J. Cell Physiol. 233, 2279-2291 (2018).

12. Zhou, Y. et al. Aquaporin 3 promotes the stem-like properties of gastric

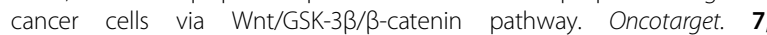
16529-16541 (2016).

13. Juuti-Uusitalo, K. et al. Aquaporin expression and function in human pluripotent stem cell-derived retinal pigmented epithelial cells. Invest. Ophthalmol. Vis. Sci. 54, 3510-3519 (2013).

14. Chen, G., Shi, Y., Liu, M. \& Sun, J. circHIPK3 regulates cell proliferation and migration by sponging miR-124 and regulating AQP3 expression in hepatocellular carcinoma. Cell. Death Dis. 9, 175 (2018).

15. Won, C. et al. Signal transducer and activator of transcription 3-mediated CD133 up-regulation contributes to promotion of hepatocellular carcinoma Hepatology. 62, 1160-1173 (2015).

16. Zhou, L. et al. Edmondson-Steiner grading increases the predictive efficiency of TNM staging for long-term survival of patients with hepatocellular carcinoma after curative resection. World J Surg. 32, 1748-1756 (2008).

17. Thomsen, R., Sølvsten, C. A., Linnet, T. E., Blechingberg, J. \& Nielsen, A. L. Analysis of $\mathrm{qPCR}$ data by converting exponentially related $\mathrm{Ct}$ values into linearly related X0 values. J. Bioinform. Comput. Biol. 8, 885-900 (2010).

18. $\mathrm{Wu}, \mathrm{G}$. et al. miR-372 down-regulates the oncogene ATAD2 to influence hepatocellular carcinoma proliferation and metastasis. BMC Cancer 14, 107 (2014).

19. Goodell, M. A. Stem cell identification and sorting using the Hoechst 33342 side population (SP). Curr. Protoc. Cytom. Chapter 9, Unit9.18 (2005).

20. Liu, Y. et al. Cancer Stem Cells are Regulated by STAT3 Signalling in Wilms Tumour. J Cancer. 9, 1486-1499 (2018).

21. Li, W., Lee, M. R., Kim, T., Kim, Y. W. \& Cho, M. Y. Activated STAT3 may participate in tumor progression through increasing CD133/survivin expression in early stage of colon cancer. Biochem. Biophys. Res. Commun. 497, 354-361 (2018).
22. Long, J. et al. Maintenance of stemness by miR-589-5p in hepatocellular carcinoma cells promotes chemoresistance via STAT3 signaling. Cancer Lett 423, 113-126 (2018)

23. Tang, K. H. et al. CD133( + ) liver tumor-initiating cells promote tumor angiogenesis, growth, and self-renewal through neurotensin/interleukin-8/ CXCL1 signaling. Hepatology 55, 807-820 (2012).

24. Kohga, K. et al. Expression of CD133 confers malignant potential by regulating metalloproteinases in human hepatocellular carcinoma. J Hepatol. 52 872-879 (2010)

25. Wang, $X$. et al. Insufficient radiofrequency ablation promotes hepatocellular carcinoma cell progression via autophagy and the CD133 feedback loop. Oncol. Rep. 40, 241-251 (2018).

26. Jang, J. W. et al. CD133 confers cancer stem-like cell properties by stabilizing EGFR-AKT signaling in hepatocellular carcinoma. Cancer Lett. 389, 1-10 (2017).

27. Bai, $\mathrm{H}$. Y. et al. Eukaryotic initiation factor $5 \mathrm{~A} 2$ contributes to the maintenance of CD133(+) hepatocellular carcinoma cells via the c-Myc/microRNA-29b Axis. Stem Cells 36, 180-191 (2018).

28. Chan, A. W., Tong, J. H., Chan, S. L., Lai, P. B. \& To, K. F. Expression of stemness markers (CD133 and EpCAM) in prognostication of hepatocellular carcinoma. Histopathology 64, 935-950 (2014).

29. Terai, K. et al. A novel oncolytic herpes capable of cell-specific transcriptional targeting of CD133 \pm cancer cells induces significant tumor regression. Stem Cells 36,1154-1169 (2018).

30. Chen, Y. L. et al. The effects of the location of cancer stem cell marker CD133 on the prognosis of hepatocellular carcinoma patients. BMC Cancer 17, 474 (2017).

31. Chen, J. et al. Aquaporin 3 promotes epithelial-mesenchymal transition in gastric cancer. J. Exp. Clin. Cancer Res. 33, 38 (2014).

32. Kang, B. W. et al. Expression of aquaporin-1, aquaporin-3, and aquaporin-5 correlates with nodal metastasis in colon cancer. Oncology 88, 369-376 (2015).

33. Li, B., Jin, L., Zhong, K. \& Du, D. Correlation of aquaporin 3 expression with the clinicopathologic characteristics of non-small cell lung cancer. Zhongguo Fei Ai Za Zhi. 15, 404-408 (2012).

34. Satooka, H. \& Hara-Chikuma, M. Aquaporin-3 controls breast cancer cel migration by regulating hydrogen peroxide transport and its downstream cell signaling. Mol. Cell Biol. 36, 1206-1218 (2016).

35. Guo, X. et al. Prognostic value of combined aquaporin 3 and aquaporin 5 overexpression in hepatocellular carcinoma. Biomed. Res. Int. 2013, 206525 (2013).

36. Ghoshal, S., Fuchs, B. C. \& Tanabe, K. K. STAT3 is a key transcriptional regulator of cancer stem cell marker CD133 in HCC. Hepatobiliary Surg. Nutr. 5, 201-203 (2016).

37. Wang, $C$. et al. Hepatitis B virus $X(\mathrm{HBX})$ induces tumorigenicity of hepatic progenitor cells in 3,5-diethoxycarbonyl-1,4-dihydrocollidine-treated HBx transgenic mice. Hepatology 55, 108-120 (2012).

38. Darnell, J. E. Jr. Transcription factors as targets for cancer therapy. Nat. Rev. Cancer 2, 740-749 (2002).

39. Hirano, T., Ishihara, K. \& Hibi, M. Roles of STAT3 in mediating the cell growth, differentiation and survival signals relayed through the IL- 6 family of cytokine receptors. Oncogene 19, 2548-2556 (2000). 